\title{
Spontaneous symmetry breaking and the Goldstone theorem in non-Hermitian field theories
}

\author{
Jean Alexandre, ${ }^{1, *}$ John Ellis, ${ }^{1,2,3, \dagger}$ Peter Millington, ${ }^{4, \$}$ and Dries Seynaeve ${ }^{1, \S}$ \\ ${ }^{1}$ Department of Physics, King's College London, London WC2R 2LS, United Kingdom \\ ${ }^{2}$ National Institute of Chemical Physics and Biophysics, Rävala 10, 10143 Tallinn, Estonia \\ ${ }^{3}$ Theoretical Physics Department, CERN, CH-1211 Geneva 23, Switzerland \\ ${ }^{4}$ School of Physics and Astronomy, University of Nottingham, Nottingham NG7 2RD, United Kingdom
}

(Received 29 May 2018; published 1 August 2018)

\begin{abstract}
We demonstrate the extension to parity-time $(\mathcal{P} \mathcal{T})$-symmetric field theories of the Goldstone theorem, confirming that the spontaneous appearance of a field vacuum expectation value via minimization of the effective potential in a non-Hermitian model is accompanied by a massless scalar boson. Laying a basis for our analysis, we first show how the conventional quantization of the path-integral formulation of quantum field theory can be extended consistently to a non-Hermitian model by considering $\mathcal{P} \mathcal{T}$ conjugation instead of Hermitian conjugation. The extension of the Goldstone theorem to a $\mathcal{P} \mathcal{T}$-symmetric field theory is made possible by the existence of a conserved current that does not, however, correspond to a symmetry of the non-Hermitian Lagrangian. In addition to extending the proof of the Goldstone theorem to a $\mathcal{P} \mathcal{T}$-symmetric theory, we exhibit a specific example in which we verify the existence of a massless boson at the tree and one-loop levels.
\end{abstract}

DOI: 10.1103/PhysRevD.98.045001

\section{INTRODUCTION}

The conventional formulations of quantum mechanics and quantum field theory (QFT) have generally been based on Hermitian Hamiltonians and Lagrangians, respectively. However, in recent years it has been established that one can also consistently formulate non-Hermitian, parity-time $(\mathcal{P T}$ )-symmetric quantum-mechanical models [1], and the possibility of a smooth transition between Hermitian and $\mathcal{P T}$-symmetric phases in quantum mechanics is described in Ref. [2]. Non-Hermitian QFTs have also been studied in various contexts. For example, a model with an $i \phi^{3}$ scalar interaction was studied in Refs. [3-6], and it was shown that a meaningful unbounded effective potential can be obtained in the framework of $\mathcal{P} \mathcal{T}$-symmetric QFT [7].

A $\mathcal{P} \mathcal{T}$-symmetric QFT involving a non-Hermitian fermion mass term $\mu \bar{\psi} \gamma^{5} \psi$ was introduced in Ref. [8]. This model was studied further in Ref. [9], where the existence of a conserved current was demonstrated and

\footnotetext{
*jean.alexandre@kcl.ac.uk

john.ellis@cern.ch

\#p.millington@nottingham.ac.uk

dries.seynaeve@kcl.ac.uk
}

Published by the American Physical Society under the terms of the Creative Commons Attribution 4.0 International license. Further distribution of this work must maintain attribution to the author(s) and the published article's title, journal citation, and DOI. Funded by SCOAP. shown to ensure the consistency of $\mathcal{P} \mathcal{T}$ symmetry with unitarity. This non-Hermitian mass term has been used for alternative descriptions of neutrino masses $[10,11]$ (see also Ref. [12] for a summary) or dark matter [13]. NonHermitian extensions of conventional QFT have also been applied to neutrino oscillations [14] and to decays of the Higgs boson [15]. Interesting studies have been done in Ref. [16] of a non-Hermitian fermionic model on the lattice, which allows for a different number of left-handed and right-handed excitations, consistent with the fermionic current density derived in Ref. [9]. We also note that the confinement phase transition in QCD has been related to $\mathcal{P T}$-symmetry properties of ghost fields in Ref. [17].

An intriguing feature of Ref. [9] was the discovery that the existence of a conserved current in a $\mathcal{P} \mathcal{T}$-symmetric QFT does not correspond to a symmetry of the Lagrangian $\mathcal{L}$, but rather to a specific transformation of $\mathcal{L}$ that is related to the non-Hermitian part of the action. $\mathcal{P} \mathcal{T}$-symmetric QFTs evade Noether's theorem [18] in the sense that symmetries of the Lagrangian do not give rise to conserved currents. Revisiting Noether's derivation, one finds that there exist conserved currents for non-Hermitiam theories, but these correspond to transformations that must effect a particular nontrivial variation of the Lagrangian, which vanishes only in the Hermitian limit. This observation raises the interesting question of whether there is an analogue in $\mathcal{P} \mathcal{T}$-symmetric QFT of spontaneous symmetry breaking and, if so, whether the breaking of a global symmetry is accompanied by a massless Goldstone mode, 
as in Hermitian QFT [19-21]. The answers provided in this paper are that the existence of a massless Goldstone mode can be shown from current conservation and does not require the Lagrangian to be invariant under the corresponding field transformation. Nevertheless, there is a symmetry of the Lagrangian, which is spontaneously broken by the choice of a specific vacuum.

However, before addressing these questions, we first discuss some basic issues in the formulation of a nonHermitian QFT, which require a consistent procedure for quantization of the path integral. This is based on the existence of a complete set of real energy eigenstates, which allow the introduction of a saddle point about which the integration of quantum fluctuations is well defined. To this end, we show how this conventional quantization of the path integral can be extended consistently to a non-Hermitian scalar QFT by considering $\mathcal{P} \mathcal{T}$ conjugation instead of Hermitian conjugation. We perform the calculation of the one-loop effective action explicitly for a generic case and, assuming that the only source of nonHermiticity is a mass term, we show that the theory is asymptotically Hermitian.

We then prove an extension of the Goldstone theorem for this non-Hermitian QFT, showing that the spontaneous appearance of a field vacuum expectation value via minimization of the effective potential is accompanied by the appearance of a massless scalar mode, whose existence is linked to the presence of a conserved current in this $\mathcal{P} \mathcal{I}$-symmetric QFT. We confirm the existence of the massless Goldstone mode by explicit calculations at both the tree and one-loop levels.

The layout of this paper is as follows. In Sec. II, we review the variational procedure (originally described in Ref. [22] and summarized in Ref. [23]) for the complex scalar model that forms the focus of this work. We also recall how the existence of a conserved current does not correspond to a symmetry of the Lagrangian $\mathcal{L}$ [9]. As we explain, a detailed study of the $\mathcal{P} \mathcal{T}$-symmetry properties of the model is required in order to understand its consistency. We then introduce in Sec. III a procedure for path-integral quantization, which is based on the existence of a complete set of eigenstates with real energies in the $\mathcal{P} \mathcal{T}$-symmetric phase of the model. We then introduce an extension of the concept of a saddle point and show that the integration of quantum fluctuations about this configuration is well defined. Finally, in Sec. IV, we discuss the extension of the Goldstone theorem to the $\mathcal{P} \mathcal{T}$-symmetric case, which follows the same steps as in an Hermitian theory, provided one considers $\mathcal{P} \mathcal{T}$-conjugate instead of Hermitianconjugate states. A summary and discussion of outstanding issues are given in Sec. V.

\section{COMPLEX SCALAR MODEL}

We consider a theory containing two complex scalar fields with the Lagrangian density

$$
\begin{aligned}
\mathcal{L}= & \partial_{\nu} \phi_{1}^{\star} \partial^{\nu} \phi_{1}+\partial_{\nu} \phi_{2}^{\star} \partial^{\nu} \phi_{2}-m_{1}^{2}\left|\phi_{1}\right|^{2}-m_{2}^{2}\left|\phi_{2}\right|^{2} \\
& -\mu^{2}\left(\phi_{1}^{\star} \phi_{2}-\phi_{2}^{\star} \phi_{1}\right)-U_{\text {int }},
\end{aligned}
$$

in which the interaction potential $U_{\text {int }}$ is $\mathcal{P} \mathcal{T}$ symmetric. The free part of this Lagrangian describes the simplest scalar model that contains a non-Hermitian but $\mathcal{P T}$-symmetric mass term [22]. The corresponding Hamiltonian is invariant under the combined action of the following $\mathcal{P}$ and $\mathcal{T}$ transformations:

$$
\begin{gathered}
\mathcal{P}: \phi_{1}(t, \mathbf{x}) \rightarrow \phi_{1}^{\prime}(t,-\mathbf{x})=+\phi_{1}(t, \mathbf{x}), \\
\phi_{2}(t, \mathbf{x}) \rightarrow \phi_{2}^{\prime}(t,-\mathbf{x})=-\phi_{2}(t, \mathbf{x}), \\
\mathcal{T}: \phi_{1}(t, \mathbf{x}) \rightarrow \phi_{1}^{\prime}(-t, \mathbf{x})=\phi_{1}^{\star}(t, \mathbf{x}), \\
\phi_{2}(t, \mathbf{x}) \rightarrow \phi_{2}^{\prime}(-t, \mathbf{x})=\phi_{2}^{\star}(t, \mathbf{x}) .
\end{gathered}
$$

Restricting our attention to the free part of the Lagrangian, it is convenient to introduce the doublet

$$
\Phi(x) \equiv\left(\begin{array}{l}
\phi_{1}(x) \\
\phi_{2}(x)
\end{array}\right)
$$

The $\mathcal{P}$ and $\mathcal{T}$ transformations can then be written in the condensed forms

$$
\begin{aligned}
& \mathcal{P}: \Phi(t, \mathbf{x}) \rightarrow \Phi^{\prime}(t,-\mathbf{x})=P \Phi(t, \mathbf{x}), \\
& \mathcal{T}: \Phi(t, \mathbf{x}) \rightarrow \Phi^{\prime}(-t, \mathbf{x})=T \Phi^{\star}(t, \mathbf{x}),
\end{aligned}
$$

where $T \equiv \operatorname{diag}(1,1)$ and $P \equiv \operatorname{diag}(1,-1)$. We note that $\phi_{1}$ transforms as a scalar and $\phi_{2}$ transforms as a pseudoscalar.

We can introduce the $\mathcal{P} \mathcal{T}$ adjoint [22] of $\Phi(x): \Phi^{\ddagger}(x) \equiv$ $\left[\Phi^{\mathcal{P T}}(x)\right]^{\top}$, where the superscript $\mathrm{T}$ indicates the matrix transpose. Neglecting total derivatives (see below), the Lagrangian density can then be written as

$$
\mathcal{L}=\Phi^{\ddagger}\left(\begin{array}{cc}
-\square-m_{1}^{2} & -\mu^{2} \\
-\mu^{2} & \square+m_{2}^{2}
\end{array}\right) \Phi-U_{\text {int }} .
$$

The variation of the action due to variations in $\Phi$ and $\Phi^{\ddagger}$ is

$$
\begin{aligned}
\delta S= & \int \mathrm{d}^{4} x\left[\left(\frac{\partial \mathcal{L}}{\partial \Phi}-\partial_{\nu} \frac{\partial \mathcal{L}}{\partial\left(\partial_{\nu} \Phi\right)}\right) \delta \Phi\right. \\
& +\delta \Phi^{\ddagger}\left(\frac{\partial \mathcal{L}}{\partial \Phi^{\ddagger}}-\partial_{\nu} \frac{\partial \mathcal{L}}{\partial\left(\partial_{\nu} \Phi^{\ddagger}\right)}\right) \\
& \left.+\partial_{\nu}\left(\frac{\partial \mathcal{L}}{\partial\left(\partial_{\nu} \Phi\right)} \delta \Phi+\delta \Phi^{\ddagger} \frac{\partial \mathcal{L}}{\partial\left(\partial_{\nu} \Phi^{\ddagger}\right)}\right)\right],
\end{aligned}
$$

and we can quickly convince ourselves that the standard Euler-Lagrange equations 


$$
\frac{\partial \mathcal{L}}{\partial \Phi}-\partial_{\nu} \frac{\partial \mathcal{L}}{\partial\left(\partial_{\nu} \Phi\right)}=0 \text { and } \frac{\partial \mathcal{L}}{\partial \Phi^{\ddagger}}-\partial_{\nu} \frac{\partial \mathcal{L}}{\partial\left(\partial_{\nu} \Phi^{\ddagger}\right)}=0
$$

are inconsistent as a result of the non-Hermiticity. Thus, if we require $\delta S=0$, the support of nontrivial solutions $\Phi \neq 0$ would require the surface terms in the second line of Eq. (6) to be nonvanishing. Alternatively, we can introduce an external source [22]. Whichever course is taken, we can choose to fix the variational procedure with respect to either $\Phi$ or $\Phi^{\ddagger}$, i.e., we can take

$$
\begin{aligned}
\frac{\delta S}{\delta \Phi} & \equiv \frac{\partial \mathcal{L}}{\partial \Phi}-\partial_{\nu} \frac{\partial \mathcal{L}}{\partial\left(\partial_{\nu} \Phi\right)}=0 \quad \text { or } \\
\frac{\delta S}{\delta \Phi^{\ddagger}} & \equiv \frac{\partial \mathcal{L}}{\partial \Phi^{\ddagger}}-\partial_{\nu} \frac{\partial \mathcal{L}}{\partial\left(\partial_{\nu} \Phi^{\ddagger}\right)}=0 .
\end{aligned}
$$

Choosing the latter, the equations of motion are

$$
\begin{aligned}
& \square \phi_{1}+m_{1}^{2} \phi_{1}+\mu^{2} \phi_{2}+\frac{\partial U_{\mathrm{int}}}{\partial \phi_{1}^{\star}}=0, \\
& \square \phi_{2}+m_{2}^{2} \phi_{2}-\mu^{2} \phi_{1}+\frac{\partial U_{\mathrm{int}}}{\partial \phi_{2}^{\star}}=0 .
\end{aligned}
$$

The squared mass eigenvalues

$$
M_{ \pm}^{2}=\frac{1}{2}\left(m_{1}^{2}+m_{2}^{2}\right) \pm \frac{1}{2} \sqrt{\left(m_{1}^{2}-m_{2}^{2}\right)^{2}-4 \mu^{4}}
$$

are real so long as we remain in the region of unbroken $\mathcal{P T}$ symmetry, requiring

$$
\eta \equiv \frac{2 \mu^{2}}{\left|m_{1}^{2}-m_{2}^{2}\right|} \leq 1 .
$$

An additional consequence of the above subtlety in the variational procedure is the way in which conserved currents arise. Having chosen to define the variational procedure with respect to $\Phi^{\ddagger}$, a careful treatment of Noether's theorem (see Ref. [22]) shows that there exists a conserved current for any transformation that satisfies

$$
\begin{aligned}
\delta \mathcal{L} & =\left(\frac{\partial \mathcal{L}}{\partial \Phi}-\partial_{\nu} \frac{\partial \mathcal{L}}{\partial\left(\partial_{\nu} \Phi\right)}\right) \delta \Phi \\
& =2 \mu^{2}\left(\phi_{2}^{\star} \delta \phi_{1}-\phi_{1}^{\star} \delta \phi_{2}\right)-2 i\left[\frac{\partial}{\partial \Phi} \operatorname{Im} U_{\text {int }}\right] \delta \Phi .
\end{aligned}
$$

Notice that $\delta \mathcal{L}=0$ in the Hermitian limit, and we recover the usual statement of Noether's theorem [18]: for every continuous symmetry of the Lagrangian, there exists a corresponding conserved current. This is not the case for our non-Hermitian theory. The two $U(1)$ currents

$$
\begin{aligned}
& j_{1}^{\nu}=i\left(\phi_{1}^{\star} \partial^{\nu} \phi_{1}-\phi_{1} \partial^{\nu} \phi_{1}^{\star}\right) \quad \text { and } \\
& j_{2}^{\nu}=i\left(\phi_{2}^{\star} \partial^{\nu} \phi_{2}-\phi_{2} \partial^{\nu} \phi_{2}^{\star}\right)
\end{aligned}
$$

are not conserved in the free theory for $\mu \neq 0$; specifically,

$$
\partial_{\nu} j_{1}^{\nu}=\partial_{\nu} j_{2}^{\nu}=i \mu^{2}\left(\phi_{2}^{\star} \phi_{1}-\phi_{1}^{\star} \phi_{2}\right) .
$$

Their difference $j^{\nu} \equiv j_{1}^{\nu}-j_{2}^{\nu}$, however, is conserved, and this current corresponds to the $U(1)$ transformations

$$
\begin{aligned}
& \phi_{1}(x) \rightarrow \phi_{1}{ }^{\prime}(x)=e^{+i \epsilon} \phi_{1}(x), \\
& \phi_{2}(x) \rightarrow \phi_{2}{ }^{\prime}(x)=e^{-i \epsilon} \phi_{2}(x),
\end{aligned}
$$

which satisfy Eq. (12) but do not leave the Lagrangian invariant. In fact, these transformations yield a oneparameter family of equivalent non-Hermitian theories whose free Lagrangians have the form

$$
\begin{aligned}
\mathcal{L}_{\epsilon}= & \partial_{\nu} \phi_{1}^{\star} \partial^{\nu} \phi_{1}+\partial_{\nu} \phi_{2}^{\star} \partial^{\nu} \phi_{2}-m_{1}^{2}\left|\phi_{1}\right|^{2}-m_{2}^{2}\left|\phi_{2}\right|^{2} \\
& -\mu^{2} e^{-2 i \epsilon} \phi_{1}^{\star} \phi_{2}+\mu^{2} e^{+2 i \epsilon} \phi_{2}^{\star} \phi_{1}
\end{aligned}
$$

and whose mass spectra are identical. That is to say, while the Lagrangian is not invariant under the transformations associated with the conserved current, physical quantities, such as the masses, are. ${ }^{1}$

Finally, we note that the definition of the vacuum is not trivial in a non-Hermitian theory, since the potential has an imaginary part. In this context, the vacuum should be defined as a solution of the equations of motion, in which case fluctuations around the vacuum have positive eigenenergies. In this section, the vacuum solution $\left(\phi_{1}, \phi_{2}\right)=(0,0)$ is symmetric, and the dispersion relations obtained above describe fluctuations above this trivial vacuum. When formulating path-integral quantization (see Sec. III), this vacuum constitutes a saddle point of the integral, around which quantum fluctuations are defined. The case of a nontrivial asymmetric solution of the equations of motion is considered in Sec. IV.

\section{PATH-INTEGRAL FORMULATION}

We now turn our attention to the formulation of the pathintegral representation of the non-Hermitian field theory.

\section{A. New conjugate field variables}

The Lagrangian in Eq. (1) would naively appear to have a finite imaginary part for $\mu \neq 0$, and one might be concerned that this could modify the convergence of the path integral. However, the spectrum of this theory is real

\footnotetext{
${ }^{1}$ Comments on the nontrivial relation between symmetries and conservation laws in non-Hermitian quantum mechanics can be found in Ref. [24].
} 
and positive definite in the region of unbroken $\mathcal{P} \mathcal{T}$ symmetry, enabling us to formulate consistently the path integral and its quantization.

We can rotate to the mass eigenbasis via the transformation

$$
\Xi \equiv R \Phi=\left(\begin{array}{l}
\xi_{1} \\
\xi_{2}
\end{array}\right), \quad \bar{\Xi} \equiv \Phi^{\dagger} R^{-1}=\left(\begin{array}{l}
\bar{\xi}_{1} \\
\bar{\xi}_{2}
\end{array}\right),
$$

where

$$
R=\mathcal{N}\left(\begin{array}{cc}
\eta & 1-\sqrt{1-\eta^{2}} \\
1-\sqrt{1-\eta^{2}} & \eta
\end{array}\right)
$$

with

$$
\mathcal{N}^{-1} \equiv \sqrt{2 \eta^{2}-2+2 \sqrt{1-\eta^{2}}}
$$

The matrix $R$ satisfies the following properties:

$$
R^{\dagger}=R, \quad R^{-1}=P R P^{-1}=P R P,
$$

such that

$\bar{\Xi}=\Xi^{\ddagger} C^{\prime}, \quad$ with $\quad \Xi^{\ddagger}=\Xi^{\dagger} P, \quad C^{\prime}=R P R^{-1}$.

The variables $\Xi$ and $\bar{\Xi}$ are $\mathcal{C}^{\prime} \mathcal{P} \mathcal{T}$-conjugate fields in the sense of Ref. [1]. We note that the $\mathcal{C}^{\prime}$ transformation here, which we identify with a prime, is not the canonical $\mathcal{C}$ transformation in Fock space, which would involve complex conjugation. Instead, it is the transformation by which one constructs the positive-definite inner product in $\mathcal{P} \mathcal{I}$-symmetric quantum mechanics [25] (see also Ref. [1]).

The free Lagrangian becomes

$\mathcal{L}_{0}=\bar{\Xi} \Delta^{-1} \Xi, \quad$ where $\Delta^{-1}=\left(\begin{array}{cc}-\square-M_{+}^{2} & 0 \\ 0 & -\square-M_{-}^{2}\end{array}\right)$,

and it appears to be that of an Hermitian theory. However, introducing interactions leads to the nontrivial feature mentioned above: varying the full action with respect to $\left(\xi_{1}, \xi_{2}\right)$ or $\left(\bar{\xi}_{1}, \bar{\xi}_{2}\right)$ does not yield the same equations of motion. This can be seen, for example, with the interaction $\left|\phi_{1} \phi_{1}^{\star}\right|^{2}$, which can be expressed using either $\Phi=R^{-1} \Xi$ :

$\left|\phi_{1} \phi_{1}^{\star}\right|^{2}=\left|\phi_{1}\right|^{4}=\mathcal{N}^{4}\left|\eta \xi_{1}+\left(\sqrt{1-\eta^{2}}-1\right) \xi_{2}\right|^{4}$,

or $\Phi^{\dagger}=\bar{\Xi} R$ :

$\left|\phi_{1} \phi_{1}^{\star}\right|^{2}=\left|\phi_{1}^{\star}\right|^{4}=\mathcal{N}^{4}\left|\eta \bar{\xi}_{1}-\left(\sqrt{1-\eta^{2}}-1\right) \bar{\xi}_{2}\right|^{4}$.

\section{B. Partition function}

The partition function is obtained from the vacuum persistence amplitude in the presence of external sources

$$
J=\left(\begin{array}{c}
j_{1} \\
j_{2}
\end{array}\right) \text { and } \bar{J}=J^{\ddagger} C^{\prime} .
$$

For the non-Hermitian theory, this vacuum persistence amplitude is

$$
Z[J, \bar{J}]=\langle\overline{0}(+\infty) \mid 0(-\infty)\rangle_{J, \bar{J}},
$$

where the state $\langle\overline{0}|$ is the $\mathcal{C}^{\prime} \mathcal{P} \mathcal{T}$ conjugate of the vacuum state. The path integral is developed in the usual way, except that one must insert complete sets of eigenstates of the Heisenberg-picture field operator $\Xi$ and its $\mathcal{C}^{\prime} \mathcal{P} \mathcal{T}$ conjugate $\bar{\Xi}$ (rather than its Hermitian conjugate) at all intermediate times. In this way, one arrives at the following result for the Euclidean path integral:

$Z[J, \bar{J}]=\int \mathcal{D}[\Xi, \bar{\Xi}] \exp \left(-S_{E}[\Xi, \bar{\Xi}]+\int_{x}(\bar{J} \Xi+\bar{\Xi} J)\right)$,

where $S_{E}$ is the Euclidean action and we use the shorthand notation $\int_{x} \equiv \int \mathrm{d}^{4} x$. Of course, having established the correct form for the partition function, one could rewrite it in terms of the original $\mathcal{P} \mathcal{T}$-conjugate variables $\Phi$ and $\Phi^{\ddagger}$ by making the change of variables and accounting for the functional Jacobian, which is nontrivial but field independent.

The partition function (27) can be expanded around the free part

$$
\begin{aligned}
Z[J, \bar{J}] & =\int \mathcal{D}[\Xi, \bar{\Xi}] \exp \left[-\int_{x} \bar{\Xi} \Delta^{-1} \Xi+\int_{x}(\bar{J} \Xi+\bar{\Xi} J)-\int_{x} U_{\text {int }}\right] \\
& =\exp \left[\int_{x} \bar{J} \Delta J\right] \int \mathcal{D}[\Pi, \bar{\Pi}] \exp \left[-\int_{x} \bar{\Pi} \Delta^{-1} \Pi-\int_{x} U_{\text {int }}\right] \\
& =\exp \left[\int_{x} \bar{J} \Delta J\right] \sum_{n=0}^{\infty} \frac{1}{n !} \int \mathcal{D}[\Pi, \bar{\Pi}] \exp \left[-\int_{x} \bar{\Pi} \Delta^{-1} \Pi\right]\left[-\int_{x} U_{\text {int }}\right]^{n},
\end{aligned}
$$


where $\Delta^{-1}=\operatorname{diag}\left(-\partial^{2}+M_{+}^{2},-\partial^{2}+M_{-}^{2}\right)$ in Euclidean signature and $\Pi \equiv \Xi-\Delta J=\left(\pi_{1}, \pi_{2}\right)^{\top}$. One can see that the perturbative structure is the usual one, comprising welldefined Gaussian integrals at each order.

\section{One-loop 1PI effective action}

There is an unambiguous definition of the classical saddle point $\left(\Xi_{0}, \bar{\Xi}_{0}\right)$ for the path integral (27), which satisfies

$$
\left[-\frac{\delta S_{E}}{\delta \Xi}+\bar{J}\right]_{0}=0=\left[-\frac{\delta S_{E}}{\delta \bar{\Xi}}+J\right]_{0}
$$

where the index 0 indicates evaluation at the configuration $\left(\Xi_{0}, \bar{\Xi}_{0}\right)$. Expanding the partition function up to quadratic order around the saddle point, we obtain for the one-loop partition function

$$
\begin{aligned}
Z^{(1)}[J, \bar{J}]= & \exp \left[-S_{E}\left[\Xi_{0}, \bar{\Xi}_{0}\right]+\int_{x}\left(\bar{J} \Xi_{0}+\bar{\Xi}_{0} J\right)\right] \\
& \times \int \mathcal{D}[\Xi, \bar{\Xi}] \exp \left[-\frac{1}{2} \int_{x y}\left(\left.2\left(\bar{\Xi}-\bar{\Xi}_{0}\right)_{x} \frac{\delta^{2} S_{E}}{\delta \bar{\Xi}_{x} \delta \Xi_{y}}\right|_{0}\left(\Xi-\Xi_{0}\right)_{y}\right.\right. \\
& \left.\left.+\left.\left(\bar{\Xi}-\bar{\Xi}_{0}\right)_{x} \frac{\delta^{2} S_{E}}{\delta \bar{\Xi}_{x} \delta \bar{\Xi}_{y}}\right|_{0}\left(\bar{\Xi}-\bar{\Xi}_{0}\right)_{y}+\left.\left(\Xi-\Xi_{0}\right)_{x} \frac{\delta^{2} S_{E}}{\delta \Xi_{x} \delta \Xi_{y}}\right|_{0}\left(\Xi-\Xi_{0}\right)_{y}\right)\right] \\
= & \exp \left[-S_{E}\left[\Xi_{0}, \bar{\Xi}_{0}\right]+\int_{x}\left(\bar{J} \Xi_{0}+\bar{\Xi}_{0} J\right)-\left.\frac{1}{2} \operatorname{STr} \ln S_{E}^{(2)}\right|_{0}\right]
\end{aligned}
$$

where $S_{E}^{(2)}$ is the functional Hessian matrix (in field space) of the Euclidean action and STr indicates the trace over both coordinate and field spaces. In order to define the one-particle irreducible (1PI) effective action $\Gamma^{(1)}$, one introduces the background field $\Xi_{c}$ :

$$
\Xi_{c}=\frac{1}{Z^{(1)}} \frac{\delta Z^{(1)}}{\delta \bar{J}},
$$

which, from Eq. (30), is

$$
\begin{aligned}
\Xi_{c} & =\Xi_{0}+\int_{x}\left(-\frac{\delta S_{E}}{\delta \Xi_{0}}+\bar{J}\right) \frac{\delta \Xi_{0}}{\delta \bar{J}}-\left.\frac{1}{2} \frac{\delta}{\delta \bar{J}} \mathrm{~S} \operatorname{Tr} \ln S_{E}^{(2)}\right|_{0} \\
& =\Xi_{0}+\text { quantum corrections. }
\end{aligned}
$$

$\Gamma^{(1)}$ is then defined after inverting the relation (31) to express $\bar{J}$ as a functional of $\Xi_{c}$ :

$$
\begin{aligned}
\Gamma^{(1)}\left[\Xi_{c}, \bar{\Xi}_{c}\right] & =-\ln Z^{(1)}+\int_{x}\left(\bar{J} \Xi_{c}+\bar{\Xi}_{c} J\right) \\
& =S_{E}\left[\Xi_{c}, \bar{\Xi}_{c}\right]+\left.\frac{1}{2} \operatorname{STr} \ln S_{E}^{(2)}\right|_{c},
\end{aligned}
$$

were the index $c$ indicates evaluation in the background field configuration. The one-loop 1PI effective potential is obtained for a constant configuration $\Xi_{c}$ and is then given by

$$
U^{(1)}\left(\Xi_{c}, \bar{\Xi}_{c}\right)=U\left(\Xi_{c}, \bar{\Xi}_{c}\right)+\left.\frac{1}{2 V^{(4)}} \mathrm{S} \operatorname{Tr} \ln S_{E}^{(2)}\right|_{c},
$$

where $V^{(4)}$ is the spacetime volume. After a rotation to the original basis, which does not affect the trace, we finally obtain

$$
U^{(1)}\left(\Phi_{c}, \Phi_{c}^{\dagger}\right)=U\left(\Phi_{c}, \Phi_{c}^{\dagger}\right)+\left.\frac{1}{2 V^{(4)}} \mathrm{S} \operatorname{Tr} \ln S_{E}^{(2)}\right|_{c} .
$$

\section{Running couplings}

We consider here a bare interaction potential of the form

$$
\begin{aligned}
U_{\text {int }}^{(0)}= & \frac{g_{1}}{4}\left|\phi_{1}\right|^{4}+\frac{g_{2}}{4}\left|\phi_{2}\right|^{4}+\lambda\left|\phi_{1} \phi_{2}\right|^{2} \\
& +\frac{\alpha}{4}\left(\left(\phi_{1}^{\star} \phi_{2}\right)^{2}+\left(\phi_{2}^{\star} \phi_{1}\right)^{2}\right) \\
& +\frac{1}{2}\left(\beta_{1}\left|\phi_{1}\right|^{2}+\beta_{2}\left|\phi_{2}\right|^{2}\right)\left(\phi_{1}^{\star} \phi_{2}-\phi_{2}^{\star} \phi_{1}\right) .
\end{aligned}
$$

Substituting this potential into Eq. (35) leads to the following one-loop running of the coupling constants (details can be found in the Appendix):

$$
\begin{aligned}
& \left(m_{i}^{2}\right)^{(1)}=m_{i}^{2}+\frac{g_{i}+\lambda}{16 \pi^{2}} \Lambda^{2}+\mathcal{O}\left(\ln \left(\frac{\Lambda}{m}\right)\right), \\
& \left(\mu^{2}\right)^{(1)}=\mu^{2}+\frac{\beta_{1}+\beta_{2}}{16 \pi^{2}} \Lambda^{2}-\frac{1}{8 \pi^{2}}\left(\mu^{2}(\lambda-\alpha)+\beta_{1} m_{1}^{2}+\beta_{2} m_{2}^{2}\right) \ln \left(\frac{\Lambda}{m}\right),
\end{aligned}
$$




$$
\begin{aligned}
& g_{i}^{(1)}=g_{i}-\frac{1}{16 \pi^{2}}\left(5 g_{i}^{2}+\alpha^{2}+4 \lambda^{2}-10 \beta_{i}^{2}\right) \ln \left(\frac{\Lambda}{m}\right), \\
& \lambda^{(1)}=\lambda-\frac{1}{16 \pi^{2}}\left(4 \lambda^{2}+2 \alpha^{2}+2 \lambda\left(g_{1}+g_{2}\right)-3\left(\beta_{1}^{2}+\beta_{2}^{2}\right)-4 \beta_{1} \beta_{2}\right) \ln \left(\frac{\Lambda}{m}\right), \\
& \alpha^{(1)}=\alpha-\frac{1}{16 \pi^{2}}\left(4\left(\beta_{1}^{2}+\beta_{2}^{2}\right)+\alpha\left(g_{1}+g_{2}\right)+2 \beta_{1} \beta_{2}+8 \lambda \alpha\right) \ln \left(\frac{\Lambda}{m}\right), \\
& \beta_{i}^{(1)}=\beta_{i}-\frac{1}{16 \pi^{2}}\left(5 g_{i} \beta_{i}+4 \beta_{j} \lambda-\alpha \beta_{j}+6 \lambda \beta_{i}-4 \alpha \beta_{i}\right) \ln \left(\frac{\Lambda}{m}\right),
\end{aligned}
$$

where $m$ is a typical mass scale of the system, $i \neq j$, and finite terms are omitted.

\section{E. Hermitian fixed point}

We assume here that the non-Hermitian interactions are switched off $\left(\beta_{i}=0\right)$ and the only source of non-Hermiticity is the mass parameter $\mu^{2}$. Quantum corrections modify this mass parameter, and we need to check that the condition (11), which delineates the phase of unbroken $\mathcal{P} \mathcal{T}$ symmetry, remains valid at one loop. For a fixed set of dressed parameters, the one-loop running of the parameter $\eta$ is

$\eta(\Lambda)=\left|\frac{\left.2\left(\mu^{2}\right)^{(1)}-\left(\alpha^{(1)}-\lambda^{(1)}\right) \mu^{2} /\left(4 \pi^{2}\right) \ln (\Lambda / m)\right)}{\left(m_{1}^{2}\right)^{(1)}-\left(m_{2}^{2}\right)^{(1)}-\left(g_{1}^{(1)}-g_{2}^{(1)}\right) \Lambda^{2} /\left(16 \pi^{2}\right)}\right|$.

We recall that, for the $\mathcal{P} \mathcal{T}$ symmetry to be unbroken, the following requirement needs to be satisfied for all values of $\Lambda$ :

$$
\eta(\Lambda)<1
$$

If $g_{1}^{(1)} \neq g_{2}^{(1)}$, we can see that $\eta(\Lambda) \rightarrow 0$ when $\Lambda \rightarrow \infty$, such that the theory converges to a Hermitian limit, which thus appears as an UV fixed point.

\section{GOLDSTONE MODES}

Having established a consistent formulation of the nonHermitian path integral and its quantization, we show, in this section, that the usual proof for the presence of Goldstone modes is still valid in the $\mathcal{P} \mathcal{T}$-symmetric case, and we explicitly derive these modes at one-loop order. As explained below, the existence of a Goldstone mode relies on a conserved current and not on the invariance of the Lagrangian. We note, however, that both are related: in the model (1), current conservation arises from the field transformation $\Phi \rightarrow \exp (i \epsilon P) \Phi$, whereas the Lagrangian is invariant under the transformation $\Phi \rightarrow \exp (i \epsilon) \Phi$. The Goldstone mode is a consequence of the former transformation, but the choice of a specific vacuum spontaneously breaks the latter symmetry.

\section{A. Proof of the Goldstone theorem}

Before considering our specific example, we first revisit the derivation of the Goldstone theorem [19-21] in the context of a non-Hermitian theory. We assume that there exists an infinitesimal transformation, which takes the generic form

$$
\Phi \rightarrow \Phi+i \epsilon T \Phi
$$

where $T$ is the generator of the transformation. We also assume that this transformation corresponds to a conserved current $j^{\nu}$ with conserved charge $Q=\int \mathrm{d}^{3} \mathbf{x} j^{0}(x)$. Most importantly, for the non-Hermitian theory, this transformation does not leave the Lagrangian invariant.

We are interested in the vacuum expectation of the commutator $[Q, \Phi(x)]$ :

$$
\langle\overline{0}|[Q, \Phi(x)]| 0\rangle=i T\langle\Phi\rangle,
$$

where $\langle\Phi\rangle \equiv\langle\overline{0}|\Phi(x)| 0\rangle$. We note that the inner product is defined with respect to $\mathcal{C}^{\prime} \mathcal{P} \mathcal{T}$, as is necessary for a nonHermitian theory. With this exception, the proof of the Goldstone theorem proceeds in the same manner as for Hermitian theories (and we closely follow Ref. [26]). By inserting complete sets of intermediate states, we can write

$$
\begin{aligned}
\left\langle\overline{0}\left|\left[j^{\nu}(y), \Phi(x)\right]\right| 0\right\rangle= & \sum_{N}\left[\left\langle\overline{0}\left|j^{\nu}(y)\right| N\right\rangle\langle\bar{N}|\Phi(x)| 0\rangle-\langle\overline{0}|\Phi(x)| N\rangle\left\langle\bar{N}\left|j^{\nu}(y)\right| 0\right\rangle\right] \\
= & \int \frac{\mathrm{d}^{4} p}{(2 \pi)^{4}} e^{-i p \cdot(y-x)} \sum_{N}\left[(2 \pi)^{4} \delta^{4}\left(p_{N}-p\right)\left\langle\overline{0}\left|j^{\nu}(0)\right| N\right\rangle\langle\bar{N}|\Phi(0)| 0\rangle\right. \\
& \left.-(2 \pi)^{4} \delta^{4}\left(p_{N}+p\right)\langle\overline{0}|\Phi(0)| N\rangle\left\langle\bar{N}\left|j^{\nu}(0)\right| 0\right\rangle\right],
\end{aligned}
$$


and, by virtue of Lorentz invariance, we have that

$$
\begin{aligned}
& \sum_{N}(2 \pi)^{4} \delta^{4}\left(p_{N}-p\right)\left\langle\overline{0}\left|j^{\nu}(0)\right| N\right\rangle\langle\bar{N}|\Phi(0)| 0\rangle=2 \pi i \theta\left(+p_{0}\right) p^{\nu} \rho\left(p^{2}\right), \\
& \sum_{N}(2 \pi)^{4} \delta^{4}\left(p_{N}+p\right)\langle\overline{0}|\Phi(0)| N\rangle\left\langle\bar{N}\left|j^{\nu}(0)\right| 0\right\rangle=2 \pi i \theta\left(-p_{0}\right) p^{\nu} \bar{\rho}\left(p^{2}\right) .
\end{aligned}
$$

Moreover, causality requires that the commutator vanish for spacelike separations, and it follows that $\rho\left(p^{2}\right)=\bar{\rho}\left(p^{2}\right)$. We then arrive at the (Källén-Lehmann) spectral representation

$$
\begin{aligned}
\left\langle\overline{0}\left|\left[j^{\nu}(y), \Phi(x)\right]\right| 0\right\rangle & =i \int \frac{\mathrm{d}^{4} p}{(2 \pi)^{4}} e^{-i p \cdot(y-x)} 2 \pi \operatorname{sgn}\left(p_{0}\right) p^{\nu} \rho\left(p^{2}\right) \\
& =-\frac{\partial}{\partial y_{\nu}} \int \mathrm{d} \sigma^{2} \rho\left(\sigma^{2}\right) \Delta\left(y, x ; \sigma^{2}\right),
\end{aligned}
$$

where

$\Delta\left(y, x ; \sigma^{2}\right)=\int \frac{\mathrm{d}^{4} p}{(2 \pi)^{4}} e^{-i p \cdot(y-x)} 2 \pi \operatorname{sgn}\left(p_{0}\right) \delta\left(p^{2}-\sigma^{2}\right)$,

is the Pauli-Jordan function with the mass of the field replaced by $\sigma$.

Since the current is conserved, it follows that

$$
\begin{aligned}
- & \square_{y} \int \mathrm{d} \sigma^{2} \rho\left(\sigma^{2}\right) \Delta\left(y, x ; \sigma^{2}\right) \\
& =\int \mathrm{d} \sigma^{2} \sigma^{2} \rho\left(\sigma^{2}\right) \Delta\left(y, x ; \sigma^{2}\right)=0,
\end{aligned}
$$

in which case $\rho\left(\sigma^{2}\right)$ must be zero for $\sigma^{2} \neq 0$, i.e., $\rho\left(\sigma^{2}\right)=\rho_{0} \delta\left(\sigma^{2}\right)$. Thus, for $x_{0}=y_{0}$, we have

$$
\left\langle\overline{0}\left|\left[j^{0}(y), \Phi(x)\right]\right| 0\right\rangle=i \rho_{0} \delta^{3}(\mathbf{y}-\mathbf{x}),
$$

and it follows that

$$
\langle\overline{0}|[Q, \Phi(x)]| 0\rangle=i T\langle\Phi\rangle=i \rho_{0} .
$$

If there exists a nontrivial vacuum $\langle\Phi\rangle$, which is not invariant under the transformation generated by $T$, then $\rho_{0} \neq 0$. We remark that, for a non-Hermitian theory, $\langle\Phi\rangle^{\prime}=$ $T\langle\Phi\rangle$ is a vacuum state of the transformed Lagrangian, e.g., for the transformations in Eq. (15), $\langle\Phi\rangle^{\prime}$ is the vacuum state of the Lagrangian in Eq. (16). The latter fact does not, however, affect the derivation of the Goldstone theorem. Returning to the expressions in Eq. (43), we have

$$
\begin{aligned}
& \sum_{N}(2 \pi)^{4} \delta^{4}\left(p_{N}-p\right)\left\langle\overline{0}\left|j^{\nu}(0)\right| N\right\rangle\langle\bar{N}|\Phi(0)| 0\rangle \\
& \quad=2 \pi i \theta\left(+p_{0}\right) p^{\nu} \rho_{0} \delta\left(p^{2}\right) .
\end{aligned}
$$

The right-hand side is nonvanishing when $p^{2}=0$, provided $p^{\nu} \neq 0^{\nu}$. It follows that there must exist a state $|N\rangle$ with $p_{N}=p$, such that $p_{N}^{2}=0$, i.e., there must exist a massless state.

We emphasize that this proof of the existence of a massless Goldstone mode relies on the existence of a conserved current and not on invariance of the Lagrangian. Hence, the Goldstone theorem persists for the nonHermitian theory, and we give further details for our specific model in what follows.

\section{B. Spontaneous symmetry breaking}

In order to study spontaneous symmetry breaking, we consider the Lagrangian (1) with $U_{\text {int }}=g\left|\phi_{1}\right|^{4} / 4$ and change the sign of the $m_{1}^{2}$ mass term, i.e.,

$$
\begin{aligned}
\mathcal{L}= & \partial_{\nu} \phi_{1}^{\star} \partial^{\nu} \phi_{1}+\partial_{\nu} \phi_{2}^{\star} \partial^{\nu} \phi_{2}+m_{1}^{2}\left|\phi_{1}\right|^{2}-m_{2}^{2}\left|\phi_{2}\right|^{2} \\
& -\mu^{2}\left(\phi_{1}^{\star} \phi_{2}-\phi_{2}^{\star} \phi_{1}\right)-\frac{g}{4}\left|\phi_{1}\right|^{4},
\end{aligned}
$$

which allows for a nontrivial vacuum structure: the vacuum expectation values are the solutions of the equations

$$
\begin{aligned}
& \frac{\delta U}{\delta \phi_{1}^{\star}}=\frac{g}{2}\left|\phi_{1}\right|^{2} \phi_{1}-m_{1}^{2} \phi_{1}+\mu^{2} \phi_{2}=0, \\
& \frac{\delta U}{\delta \phi_{2}^{\star}}=m_{2}^{2} \phi_{2}-\mu^{2} \phi_{1}=0,
\end{aligned}
$$

conditions similar to the equations of motion. These equations are invariant under a phase transformation acting identically on both the fields. The nontrivial solutions to these equations are given by

$$
\left(\begin{array}{l}
v_{1} \\
v_{2}
\end{array}\right)=\sqrt{2 \frac{m_{1}^{2} m_{2}^{2}-\mu^{4}}{g m_{2}^{2}}}\left(\begin{array}{c}
1 \\
\frac{\mu^{2}}{m_{2}^{2}}
\end{array}\right) e^{i \epsilon} .
$$

For a fixed phase $\epsilon$, we can express the fields as fluctuations around these vacua, 


$$
\phi_{1}=v_{1}+\hat{\phi}_{1} \quad \text { and } \quad \phi_{2}=v_{2}+\hat{\phi}_{2}
$$

Expressing the equations of motion (9) in terms of the field fluctuations $\hat{\phi}_{1,2}$ gives

$$
\begin{aligned}
\square\left(\begin{array}{c}
\hat{\phi}_{1} \\
\hat{\phi}_{1}^{\star} \\
\hat{\phi}_{2} \\
\hat{\phi}_{2}^{\star}
\end{array}\right)= & \left(\begin{array}{cccc}
\frac{\left(m_{1}^{2} m_{2}^{2}-2 \mu^{4}\right)}{m_{2}^{2}} & \frac{\left(m_{1}^{2} m_{2}^{2}-\mu^{4}\right)}{m_{2}^{2}} & \mu^{2} & 0 \\
\frac{\left(m_{1}^{2} m_{2}^{2}-\mu^{4}\right)}{m_{2}^{2}} & \frac{\left(m_{1}^{2} m_{2}^{2}-2 \mu^{4}\right)}{m_{2}^{2}} & 0 & \mu^{2} \\
-\mu^{2} & 0 & m_{2}^{2} & 0 \\
0 & -\mu^{2} & 0 & m_{2}^{2}
\end{array}\right)\left(\begin{array}{c}
\hat{\phi}_{1} \\
\hat{\phi}_{1}^{\star} \\
\hat{\phi}_{2} \\
\hat{\phi}_{2}^{\star}
\end{array}\right) \\
& +\cdots,
\end{aligned}
$$

where the dots represent terms of higher order in $\hat{\phi}_{1}$ and $\hat{\phi}_{1}^{\star}$.

It is easy to check that the determinant of this mass matrix is zero, and we therefore have the anticipated Goldstone mode. We remark that, while the explicit forms of the eigenmodes depend on the choice of the equations of motion, the eigenspectrum is unique.

The mass matrix has a single zero eigenvalue, and the corresponding (Goldstone) mode is

$$
G_{1}=\sqrt{\frac{2 m_{2}^{4}}{m_{2}^{4}+\mu^{4}}}\left(\operatorname{Im} \hat{\phi}_{1}-\frac{\mu^{2}}{m_{2}^{2}} \operatorname{Im} \hat{\phi}_{2}\right) .
$$

For completeness, we list the other eigenvalues and their corresponding eigenmodes:

$$
\begin{aligned}
& \lambda_{2}=m_{2}^{2}-\frac{\mu^{4}}{m_{2}^{2}}, \\
& \lambda_{3}=\frac{1}{2 m_{2}^{2}}\left(2 m_{1}^{2} m_{2}^{2}-3 \mu^{4}+m_{2}^{4}+\sqrt{\left(2 m_{1}^{2} m_{2}^{2}-3 \mu^{4}-m_{2}^{4}\right)^{2}-4 \mu^{4} m_{2}^{4}}\right), \\
& \lambda_{4}=\frac{1}{2 m_{2}^{2}}\left(2 m_{1}^{2} m_{2}^{2}-3 \mu^{4}+m_{2}^{4}-\sqrt{\left(2 m_{1}^{2} m_{2}^{2}-3 \mu^{4}-m_{2}^{4}\right)^{2}-4 \mu^{4} m_{2}^{4}}\right),
\end{aligned}
$$

with

$$
\begin{aligned}
G_{2} & =\sqrt{\frac{2 m_{2}^{4}}{m_{2}^{4}+\mu^{4}}}\left(\operatorname{Im} \hat{\phi}_{2}-\frac{\mu^{2}}{m_{2}^{2}} \operatorname{Im} \hat{\phi}_{1}\right), \\
G_{3} & =\sqrt{2}\left[1+\left(\frac{\mu^{2}}{\lambda_{3}-m_{2}^{2}}\right)^{2}\right]^{-1 / 2}\left[\operatorname{Re} \hat{\phi}_{1}+\left(\frac{\mu^{2}}{\lambda_{3}-m_{2}^{2}}\right) \operatorname{Re} \hat{\phi}_{2}\right], \\
G_{4} & =\sqrt{2}\left[1+\left(\frac{\lambda_{4}-m_{2}^{2}}{\mu^{2}}\right)^{2}\right]^{-1 / 2}\left[\operatorname{Re} \hat{\phi}_{2}+\left(\frac{\lambda_{4}-m_{2}^{2}}{\mu^{2}}\right) \operatorname{Re} \hat{\phi}_{1}\right] .
\end{aligned}
$$

The form of the Goldstone mode could also have been anticipated from the conserved current itself. The conservation equation yields

$\partial_{\nu} j^{\nu}=i \partial_{\nu}\left[\left(\phi_{1}^{\star} \partial^{\nu} \phi_{1}-\phi_{1} \partial^{\nu} \phi_{1}^{\star}\right)-\left(\phi_{2}^{\star} \partial^{\nu} \phi_{2}-\phi_{2} \partial^{\nu} \phi_{2}^{\star}\right)\right]=0$.

Expanding this to first order in the fluctuations [setting the constant phase in the vacuum expectation values (vev's) $v_{1}$ and $v_{2}$ to zero] gives

$$
\partial_{\nu} j^{\nu} \simeq-2\left(v_{1} \square \operatorname{Im} \hat{\phi}_{1}-v_{2} \square \operatorname{Im} \hat{\phi}_{2}\right),
$$

and we see that the Goldstone mode is

$$
G_{1} \propto \operatorname{Im} \hat{\phi}_{1}-\frac{\mu^{2}}{m_{2}^{2}} \operatorname{Im} \hat{\phi}_{2}
$$

Finally, we note that for our choice of equations of motion, the Goldstone mode is in fact the left eigenvector of the mass matrix (as dictated by the conserved current). Choosing the alternative definition of the variational procedure, the Goldstone mode would instead correspond to the right eigenvector of the mass matrix in Eq. (54), which is distinct and related to the previous one by $\mathcal{P} \mathcal{T}$ conjugation. Note that this is consistent with $\mathcal{P} \mathcal{T}$ transformation superseding Hermitian conjugation for non-Hermitian theories and that the alternative definitions are equivalent.

\section{The Goldstone mode to one-loop order}

The full tree-level potential is given in terms of the fields $\hat{\phi}_{1}$ and $\hat{\phi}_{2}$ as 


$$
\begin{aligned}
U^{(0)}= & M_{1}^{2}\left|\hat{\phi}_{1}\right|^{2}+m_{2}^{2} \hat{\phi}_{2}\left(\hat{\phi}_{2}^{\star}+M_{c}\right) \\
& +\mu^{2}\left(\hat{\phi}_{2} \hat{\phi}_{1}^{\star}-\hat{\phi}_{1}\left(\hat{\phi}_{2}^{\star}+M_{c}\right)\right) \\
& +\frac{M_{a}^{2}}{2}\left(\hat{\phi}_{1}^{2}+\left(\hat{\phi}_{1}^{\star}\right)^{2}\right)+\frac{M_{b}}{2}\left|\hat{\phi}_{1}\right|^{2}\left(\hat{\phi}_{1}^{\star}+\hat{\phi}_{1}\right)+\frac{g}{4}\left|\hat{\phi}_{1}\right|^{4},
\end{aligned}
$$

where we use the notation

$$
\begin{aligned}
& M_{1}^{2}=\frac{m_{1}^{2} m_{2}^{2}-2 \mu^{4}}{m_{2}^{2}}, \\
& M_{a}^{2}=\frac{m_{1}^{2} m_{2}^{2}-\mu^{4}}{m_{2}^{2}}=M_{1}^{2}+\frac{\mu^{4}}{m_{2}^{2}}, \\
& M_{b}=g \sqrt{2 \frac{m_{1}^{2} m_{2}^{2}-\mu^{4}}{g m_{2}^{2}}}, \\
& M_{c}=\frac{2 \mu^{2}}{m_{2}^{2}} \sqrt{2 \frac{m_{1}^{2} m_{2}^{2}-\mu^{4}}{g m_{2}^{2}}} .
\end{aligned}
$$

The linear terms in the potential are a consequence of the non-Hermitian nature of the system. At one-loop level, these couplings are obtained by substituting this potential into Eq. (35) and are given by

$$
\begin{aligned}
g^{(1)} & =g-\frac{5 g^{2}}{16 \pi^{2}} \ln \left(\frac{\Lambda}{m}\right), \\
m_{2}^{2(1)} & =m_{2}^{2} \\
\mu^{2(1)} & =\mu^{2}, \\
M_{1}^{2(1)} & =M_{1}^{2}+\frac{g \Lambda^{2}}{16 \pi^{2}}+\mathcal{O}(\ln (\Lambda / m)), \\
M_{a}^{2(1)} & =M_{a}^{2}-\frac{1}{16 \pi^{2}}\left(2 M_{b}^{2}+g M_{a}^{2}\right) \ln \left(\frac{\Lambda}{m}\right), \\
M_{b}^{(1)} & =M_{b}-\frac{5 g M_{b}}{16 \pi^{2}} \ln \left(\frac{\Lambda}{m}\right),
\end{aligned}
$$

where finite terms are again omitted. A linear term is also generated, which is given by

$$
M_{b} \frac{\Lambda^{2}}{16 \pi^{2}}\left(\hat{\phi}_{1}^{\star}+\hat{\phi}_{1}\right)
$$

so that the one-loop potential in terms of $\hat{\phi}_{1}, \hat{\phi}_{2}$ becomes

$$
\begin{aligned}
U^{(1)}= & M_{b} \frac{\Lambda^{2}}{16 \pi^{2}}\left(\hat{\phi}_{1}+\hat{\phi}_{1}^{\star}\right)+m_{2}^{2} M_{c} \hat{\phi}_{2}-\mu^{2} M_{c} \hat{\phi}_{1}+\left(M_{1}^{2}+\frac{g \Lambda^{2}}{16 \pi^{2}}\right)\left|\hat{\phi}_{1}\right|^{2}+m_{2}^{2}\left|\hat{\phi}_{2}\right|^{2} \\
& +\mu^{2}\left(\hat{\phi}_{2} \hat{\phi}_{1}^{\star}-\hat{\phi}_{1} \hat{\phi}_{2}^{\star}\right)+\left(\frac{M_{a}^{2}}{2}-\left(M_{b}^{2}+\frac{g M_{a}^{2}}{2}\right) \frac{\ln \left(\frac{\Lambda}{m}\right)}{16 \pi^{2}}\right)\left(\hat{\phi}_{1}^{2}+\left(\hat{\phi}_{1}^{\star}\right)^{2}\right) \\
& +\frac{M_{b}}{2}\left(1-\frac{5 g \ln \left(\frac{\Lambda}{m}\right)}{16 \pi^{2}}\right)\left|\hat{\phi}_{1}\right|^{2}\left(\hat{\phi}_{1}^{\star}+\hat{\phi}_{1}\right)+\frac{g}{4}\left(1-\frac{5 g \ln \left(\frac{\Lambda}{m}\right)}{16 \pi^{2}}\right)\left|\hat{\phi}_{1}\right|^{4} .
\end{aligned}
$$

To show the existence of the Goldstone mode to one-loop order, we should express the fields in terms of fluctuations around the new shifted vacuum. From this, we can find the one-loop-corrected vev's

$$
\left(\begin{array}{c}
v_{1}^{(1)} \\
v_{2}^{(1)}
\end{array}\right)=\left(1-\frac{g}{2 M_{a}^{2}} \frac{\Lambda^{2}}{16 \pi^{2}}\right)\left(\begin{array}{l}
v_{1} \\
v_{2}
\end{array}\right) .
$$

Expressing the one-loop potential in terms of the fields fluctuating around this minimum

$$
\phi_{1}=v_{1}^{(1)}+\hat{\phi}_{1}^{(1)} \text { and } \quad \phi_{2}=v_{2}^{(1)}+\hat{\phi}_{2}^{(1)}
$$

gives equations of motion of the form

$$
\square\left(\begin{array}{c}
\hat{\phi}_{1}^{(1)} \\
\left(\hat{\phi}_{1}^{(1)}\right)^{\star} \\
\hat{\phi}_{2}^{(1)} \\
\left(\hat{\phi}_{2}^{(1)}\right)^{\star}
\end{array}\right)=\left(\begin{array}{cccc}
M_{1}^{2}-\frac{g \Lambda^{2}}{16 \pi^{2}} & M_{a}^{2}-\frac{g \Lambda^{2}}{16 \pi^{2}} & \mu^{2} & 0 \\
M_{a}^{2}-\frac{g \Lambda^{2}}{16 \pi^{2}} & M_{1}^{2}-\frac{g \Lambda^{2}}{16 \pi^{2}} & 0 & \mu^{2} \\
-\mu^{2} & 0 & m_{2}^{2} & 0 \\
0 & -\mu^{2} & 0 & m_{2}^{2}
\end{array}\right)\left(\begin{array}{c}
\hat{\phi}_{1}^{(1)} \\
\left(\hat{\phi}_{1}^{(1)}\right)^{\star} \\
\hat{\phi}_{2}^{(1)} \\
\left(\hat{\phi}_{2}^{(1)}\right)^{\star}
\end{array}\right)+\cdots
$$


The mass matrix again has determinant zero, showing that we still have a Goldstone mode at one-loop order, which is given by

$$
G_{1}^{(1)}=\sqrt{\frac{2 m_{2}^{4}}{m_{2}^{4}+\mu^{4}}}\left(\operatorname{Im} \hat{\phi}_{1}^{(1)}-\frac{\mu^{2}}{m_{2}^{2}} \operatorname{Im} \hat{\phi}_{2}^{(1)}\right) .
$$

We see that the one-loop Goldstone mode is related to the Goldstone mode at tree level; the one-loop mode is obtained from the tree-level one simply by making the replacement $\hat{\phi}_{1}, \hat{\phi}_{2} \rightarrow \hat{\phi}_{1}^{(1)}, \hat{\phi}_{2}^{(1)}$.

\section{SUMMARY AND OPEN QUESTIONS}

The nature of spontaneous symmetry breaking is a fascinating and deep issue in quantum field theory. In conventional Hermitian QFT, it is well understood how the spontaneous breaking of a global symmetry is accompanied by the appearance of a massless scalar Goldstone boson. The counterpart of the Goldstone theorem in $\mathcal{P T}$-symmetric QFT presented certain puzzles and has not been known until now. The central issue was that, although $\mathcal{P} \mathcal{T}$-symmetric theories may contain conserved currents, there are no corresponding symmetries of the Lagrangian: Noether's theorem does not apply [9] in the familiar sense. One could then wonder whether or not the existence of a conserved current would be sufficient to guarantee the appearance of a Goldstone boson.

We have shown in this paper that the answer is yes: current conservation still guarantees the existence of a massless boson. We have demonstrated this formally and also at the tree and one-loop levels in a simple $\mathcal{P} \mathcal{T}$-symmetric QFT with two complex scalar fields.

In order to investigate the Goldstone theorem in a $\mathcal{P T}$-symmetric theory, we studied the formulation of the path integral and its quantization in non-Hermitian field QFT. Since a $\mathcal{P T}$-symmetric theory possesses a complete set of real energy eigenstates, its path integral contains saddle points about which the path integration of quantum fluctuations is well defined, as long as one considers $\mathcal{P} \mathcal{T}$-conjugate pairs of fields instead of Hermitian-conjugate pairs.

The analysis in this paper can be regarded as the first step in an exploration of whether there exists a consistent $\mathcal{P} \mathcal{T}$ symmetric generalization of the Standard Model and other gauge theories. In this connection, the absence of a generalization to non-Hermitian theories of Noether's theorem is a key issue. We emphasize again that, in these theories, the existence of a conserved current does not imply the existence of a corresponding symmetry. How do gauge theories react to this situation and, in particular, do they possess a "Higgs phase"? We plan to address these issues in future work.

\section{ACKNOWLEDGMENTS}

The work of J. A., J. E. and D. S. was supported by the United Kingdom Science and Technology Facilities Council (STFC) Grant No. ST/P000258/1, and that of J. E. also by the Estonian Research Council via a Mobilitas Pluss grant. The work of P. M. was supported by STFC Grant No. ST/L000393/1 and a Leverhulme Trust Research Leadership Award.

\section{APPENDIX: RUNNING COUPLINGS}

The full bare potential is

$$
\begin{aligned}
U^{(0)}= & m_{1}^{2}\left|\phi_{1}\right|^{2}+m_{2}^{2}\left|\phi_{2}\right|^{2}+\mu^{2}\left(\phi_{1}^{\star} \phi_{2}-\phi_{2}^{\star} \phi_{1}\right) \\
& +\frac{g_{1}}{4}\left|\phi_{1}\right|^{4}+\frac{g_{2}}{4}\left|\phi_{2}\right|^{4}+\lambda\left|\phi_{1} \phi_{2}\right|^{2} \\
+ & \frac{\alpha}{4}\left(\left(\phi_{1}^{\star} \phi_{2}\right)^{2}+\left(\phi_{2}^{\star} \phi_{1}\right)^{2}\right) \\
& +\frac{1}{2}\left(\beta_{1}\left|\phi_{1}\right|^{2}+\beta_{2}\left|\phi_{2}\right|^{2}\right)\left(\phi_{1}^{\star} \phi_{2}-\phi_{2}^{\star} \phi_{1}\right),
\end{aligned}
$$

and the one-loop 1PI potential is given by

$$
U^{(1)}=U^{(0)}+\frac{1}{2 V^{(4)}} \mathrm{S} \operatorname{Tr} \ln S_{E}^{(2)},
$$

where

$$
S_{E}^{(2)}=\left(\begin{array}{cccc}
p^{2}+U_{11^{\star}}^{(0)} & U_{11}^{(0)} & U_{12^{\star}}^{(0)} & U_{12}^{(0)} \\
U_{1^{\star} 1^{\star}}^{(0)} & p^{2}+U_{1^{\star} 1}^{(0)} & U_{1^{\star} 2^{\star}}^{(0)} & U_{1^{\star} 2}^{(0)} \\
U_{21^{\star}}^{(0)} & U_{21}^{(0)} & p^{2}+U_{22^{\star}}^{(0)} & U_{22}^{(0)} \\
U_{2^{\star} 1^{\star}}^{(0)} & U_{2^{\star} 1}^{(0)} & U_{2^{\star} 2^{\star}}^{(0)} & p^{2}+U_{2^{\star} 2}^{(0)}
\end{array}\right),
$$

with

$$
U_{i^{(\star)} j^{[\star]}}=\frac{\delta^{2} U}{\delta \phi_{i}^{(\star)} \delta \phi_{j}^{[\star]}} .
$$

We have then that

$$
\begin{aligned}
\frac{1}{p^{8}} \operatorname{det} S_{E}^{(2)}= & 1+\frac{2}{p^{2}}\left(U_{11^{\star}}^{(0)}+U_{22^{\star}}^{(0)}+\frac{1}{p^{4}}\left(\left(U_{11^{\star}}^{(0)}\right)^{2}+\left(U_{22^{\star}}^{(0)}\right)^{2}+4 U_{11^{\star}}^{(0)} U_{22^{\star}}^{(0)}-U_{11}^{(0)} U_{1^{\star} 1^{\star}}^{(0)}-U_{22}^{(0)} U_{2^{\star} 2^{\star}}^{(0)}-2 U_{12}^{(0)} U_{1^{\star} 2^{\star}}^{(0)}\right.\right. \\
& \left.-2 U_{12^{\star}}^{(0)} U_{1^{\star} 2}^{(0)}\right)+\mathcal{O}\left(\frac{1}{p^{6}}\right),
\end{aligned}
$$


such that, up to finite terms,

$$
\begin{aligned}
\frac{1}{2 V^{(4)}} \operatorname{STr} \ln S_{E}^{(2)}= & \frac{1}{8 \pi^{2}} \int \mathrm{d} p p\left(U_{11^{\star}}^{(0)}+U_{22^{\star}}^{(0)}\right) \\
& -\int \frac{\mathrm{d} p}{16 \pi^{2} p}\left(\left(U_{11^{\star}}^{(0)}\right)^{2}+\left(U_{22^{\star}}^{(0)}\right)^{2}+U_{11}^{(0)} U_{1^{\star} 1^{\star}}^{(0)}+U_{22}^{(0)} U_{2^{\star} 2^{\star}}^{(0)}+2 U_{12}^{(0)} U_{1^{\star} 2^{\star}}^{(0)}+2 U_{12^{\star}}^{(0)} U_{1^{\star} 2}^{(0)}\right),
\end{aligned}
$$

and substituting the potential (A1) into this expression gives the one-loop corrections (37).

[1] C. M. Bender, Introduction to $\mathcal{P} \mathcal{T}$-symmetric quantum theory, Contemp. Phys. 46, 277 (2005).

[2] M. Znojil, Hermitian-non-Hermitian interfaces in quantum theory, Adv. High Energy Phys. 2018, 7906536 (2018).

[3] M. P. Blencowe, H. F. Jones, and A. P. Korte, Applying the linear $\delta$ expansion to the $i \phi^{3}$ interaction, Phys. Rev. D 57, 5092 (1998).

[4] H. F. Jones, The $C$ operator in $i \phi^{3}$ field theory, Czech. J. Phys. 54, 1107 (2004).

[5] C. M. Bender, V. Branchina, and E. Messina, Critical behavior of the $\mathcal{P} \mathcal{T}$-symmetric $i \phi^{3}$ quantum field theory, Phys. Rev. D 87, 085029 (2013).

[6] A. M. Shalaby, Vacuum structure and $\mathcal{P} \mathcal{T}$-symmetry breaking of the non-Hermetian $\left(i \phi^{3}\right)$ theory, Phys. Rev. D 96, 025015 (2017).

[7] C. M. Bender, D. W. Hook, N.E. Mavromatos, and S. Sarkar, $\mathcal{P} \mathcal{T}$-symmetric interpretation of unstable effective potentials, J. Phys. A: Math. Theor. 49, 45LT01 (2016).

[8] C. M. Bender, H.F. Jones, and R. J. Rivers, Dual $\mathcal{P} \mathcal{T}$-symmetric quantum field theories, Phys. Lett. B 625, 333 (2005).

[9] J. Alexandre and C. M. Bender, Foldy-Wouthuysen transformation for non-Hermitian Hamiltonians, J. Phys. A: Math. Theor. 48, 185403 (2015).

[10] K. Jones-Smith and H. Mathur, Relativistic non-Hermitian quantum mechanics, Phys. Rev. D 89, 125014 (2014).

[11] J. Alexandre, C. M. Bender, and P. Millington, NonHermitian extension of gauge theories and implications for neutrino physics, J. High Energy Phys. 11 (2015) 111.

[12] J. Alexandre, C. M. Bender, and P. Millington, Light neutrino masses from a non-Hermitian Yukawa theory, J. Phys. Conf. Ser. 873, 012047 (2017).

[13] V. N. Rodionov and A. M. Mandel, An upper limit on fermion mass spectrum in non-Hermitian models and its implications for studying of dark matter, arXiv:1708.08394.
[14] T. Ohlsson, Non-Hermitian neutrino oscillations in matter with $\mathcal{P} \mathcal{T}$ symmetric Hamiltonians, Europhys. Lett. 113, 61001 (2016).

[15] A. Y. Korchin and V. A. Kovalchuk, Decay of the Higgs boson to $\tau^{-} \tau^{+}$and non-Hermiticy of the Yukawa interaction, Phys. Rev. D 94, 076003 (2016).

[16] M. N. Chernodub, The Nielsen-Ninomiya theorem, $\mathcal{P T}$-invariant non-Hermiticity and single 8-shaped Dirac cone, J. Phys. A: Math. Theor. 50, 385001 (2017).

[17] H. Raval and B. P. Mandal, Deconfinement to confinement as a $\mathcal{P} \mathcal{T}$ phase transition, arXiv:1805.02510.

[18] E. Noether, Invariant variation problems, Nachrichten von der Gesellschaft der Wissenschaften zu Göttingen, Mathematisch-Physikalische Klasse 1918, 235 (1918).

[19] Y. Nambu, Axial Vector Current Conservation in Weak Interactions, Phys. Rev. Lett. 4, 380 (1960).

[20] J. Goldstone, Field theories with superconductor solutions, Nuovo Cimento 19, 154 (1961).

[21] J. Goldstone, A. Salam, and S. Weinberg, Broken symmetries, Phys. Rev. 127, 965 (1962).

[22] J. Alexandre, P. Millington, and D. Seynaeve, Symmetries and conservation laws in non-Hermitian field theories, Phys. Rev. D 96, 065027 (2017).

[23] J. Alexandre, P. Millington, and D. Seynaeve, Consistent description of field theories with non-Hermitian mass terms, J. Phys. Conf. Ser. 952, 012012 (2018).

[24] M. A. Simón Martínez, A. Buendía, and J.G. Muga, Symmetries and invariants for non-Hermitian Hamiltonians, Mathematics 6, 111 (2018).

[25] C. M. Bender, D. C. Brody, and H. F. Jones, Complex Extension of Quantum Mechanics, Phys. Rev. Lett. 89, 270401 (2002); 92, 119902(E) (2004).

[26] E. Weinberg, Lecture notes for quantum field theory III (2011) (unpublished). 